\title{
REDUCING THE CLOCKWISE-ALGORITHM TO $k$ LENGTH CLASSES
}

\author{
Marco Ripà \\ sPIqr Society, World Intelligence Network \\ Email : marcokrt1984@yahoo.it
}

\begin{abstract}
In the present paper, we consider an optimization problem related to the extension in $k$-dimensions of the well known $3 \times 3$ points problem by Sam Loyd. In particular, thanks to a variation of the so called "clockwise-algorithm", we show how it is possible to visit all the $3^{k}$ points of the $k$-dimensional grid given by the Cartesian product of $(0,1,2)$ using covering trails formed by $h(k)=\frac{3^{k}-1}{2}$ links who belong to $k$ (Euclidean) length classes. We can do this under the additional constraint of allowing only turning points which belong to the set $B(k):=\{(0,3) \times(0,3) \times \cdots \times(0,3)\}$. In the end, we prove that the set $G_{3}:=\{(0,1,2) \times(0,1,2) \times(0,1,2)\}$ can be covered with an inside the box simple path consisting of $3^{3}-1$ edges, all belonging to the length class $\sqrt{5}$.
\end{abstract}

Keywords: Nine dots puzzle, Clockwise-algorithm, Thinking outside the box, Polygonal path, Optimization problem, Link length, Knight's tour, TSP.

\section{INTRODUCTION}

The goal of the present paper is to solve in $\mathbb{R}^{k}$ the $3^{k}$-points problem $[1,2,3]$, which is the generalization to $k$ dimensions [4] of the classic nine dots puzzle (the well known thinking outside the box challenge) $[5,6,7]$, under two additional constraints involving the $\frac{3^{k}-3}{2}$ turning points of the minimal covering trail, $C(k)$, and considering a maximum of $k$ Euclidean length classes for all its $h(k)=\frac{3^{k}-1}{2}$ links. Since the standard $3^{k}$-points problem has been already discussed in [8], we aim to solve it using a (slightly) different covering trail, $H(k)$, which has all its $h^{\prime}(k)=h(k)$ links that belong to $k$ length classes, and accepting only turning points set on the vertices of a $3 \times 3 \times \cdots \times 3$ hypercube; those $k$-cubes of (hyper)volume $3^{k}$ units $^{k}$ have been generically indicated in [8] as box, and the sets of all their vertices will be defined below as $B_{k}$.

Definition 1 Let us define, $\forall k \in \mathbb{N}-\{0\}, B_{k}:=\{(0,3) \times(0,3) \times \cdots \times(0,3)\} \subset \mathbb{N}_{0}{ }^{k}$ as $k$-box. Thus, for any $k \geq 3$, the $k$-box corresponds to the set of all the $2^{k}$ vertices of the $k$-dimensional cube $[0,3] \times[0,3] \times \cdots \times[0,3] \subset \mathbb{R}^{k}$ which has 3 units long edges and $C_{k}=\left(\frac{3}{2}, \frac{3}{2}, \ldots, \frac{3}{2}\right)$ as its center.

In order to compactly state the enhanced $3^{k}$-points problem, let us specify which set of $3^{k}$ points our optimal covering trail should join. 
Definition 2 Let us define, $\forall k \in \mathbb{N}-\{0\}, G_{k}:=\{(0,1,2) \times(0,1,2) \times \cdots \times(0,1,2)\}$ as $k$-grid, so the $k$-grid is a set of $3^{k}$ points in the Euclidean space $\mathbb{R}^{k}$.

The enhanced $3^{k}$-points problem states:

"Given the finite set of $3^{k}$ points $G_{k}$ in $\mathbb{R}^{k}$, we need to visit all of them (at least once) with a polygonal chain $H(k)$ that has the minimum number of line segments (links), $h(k)=\frac{3^{k}-1}{2}$. We are asked to join all the points of $G_{k}$ with $h(k)$ links belonging to at most $k$ different Euclidean length classes (we are referring to the Euclidean distance metric), and additionally we are only allowed to turn, once or more, at the points belonging to the set $B_{k}$ (see Definition 1)".

In Section II, we revisit the clockwise-algorithm (for a more detailed explanation, see [8]), which works as follows:

"Given $k=1$, we join 3 collinear points with a single line, remaining inside a unidimensional box which is 3 units long. For $k=2$, we are facing the classic nine dots puzzle considering a $3 \times 3$ box, and the well-known Hamiltonian path by Sam Loyd [7] proves that we can solve the problem, without allowing any line to exit from the box, if we start from any node of the grid except from the central one. Given $k=3$, as a generalization of the original Loyd's pattern (see Figure 1), we solve the problem inside a $3 \times 3 \times 3$ box following the optimal twodimensional covering trail swirling in one more dimension, according to a basic 3-steps scheme, and beginning from a congruent starting point. Thus, if we take one vertex of $G_{3}$, while we rotate in the space at every turn, it is possible to repeat twice (forward and backward) the aforementioned two-dimensional pattern; at the end of the process, $3^{3-2}-\frac{1}{3}$ gyratories have been performed, so we spend the $\left(3^{3-1}\right)$-th line to close the subtour, joining $3-1$ new points. In this way, we reach the starting vertex again, and the last $3^{3}-1$ unvisited nodes belong only to $G_{k-1}=G_{2}$ (choosing the right direction). Therefore, we can finally paste Loyd's expected solution for his nine dots puzzle by extending one unit backward the first line, in order to visit all the remaining nodes of $G_{3}$. The method to solve the 4-dimensional case is basically the same one that we have discussed for $G_{3}$ : we apply the known 3-dimensional covering trail forward (while we spin around following the 3-steps gyratory), then backward, subsequently we return to the starting vertex with line 27 , and lastly we join the $3^{3}-1$ unvisited nodes with the aforementioned three-dimensional pattern by simply extending backward its first line. Since the clockwise-algorithm takes a $(k-1)$-dimensional solution as input and returns a $k$ dimensional solution as its output, it holds for any $k \in \mathbb{N}-\{0\}$. Thus, it solves the $3^{k}$-points problem, inside a $3 \times 3 \times \cdots \times 3$ box of hyper-volume $3^{k}$ units $^{k}$, drawing optimal trails".

Considering the case $k=2$, the result is shown in Figure 1, while Section III presents the solution of a new covering path problem on the 2 -grid and 3 -grid $[9,10,11,12,13]$. 


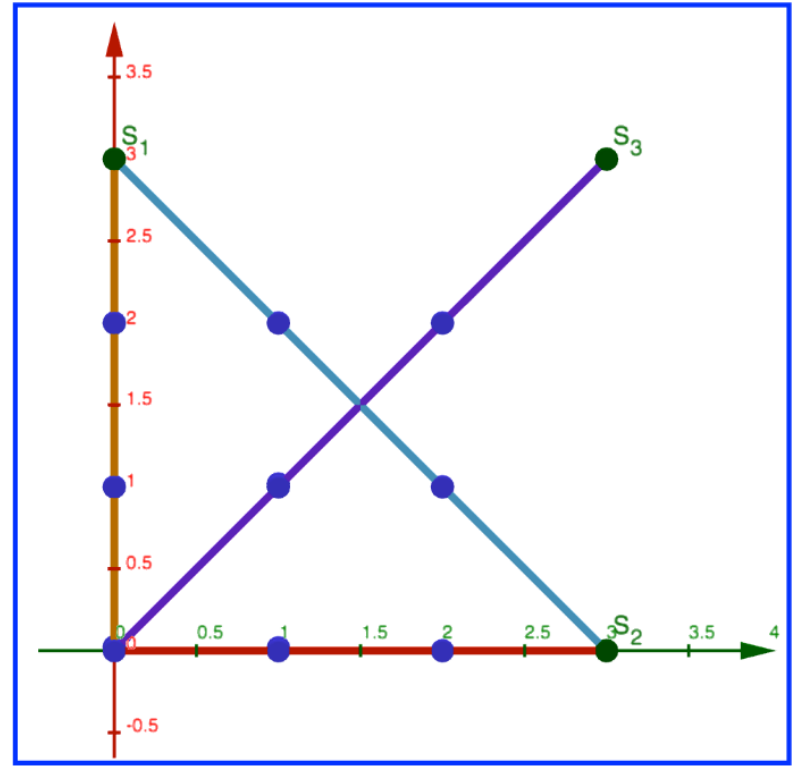

Figure 1. The extended clockwise-algorithm for the case $k=2$. Steiner points are in green (picture realized with GeoGebra [14]).

\section{MAIN RESULT}

The enhanced $3^{k}$-points problem can be optimally solved with the extended clockwisealgorithm (e.g., see Figures $2 \& 3$ for the case $k=3$ ), and this result is formally stated in Theorem 1.

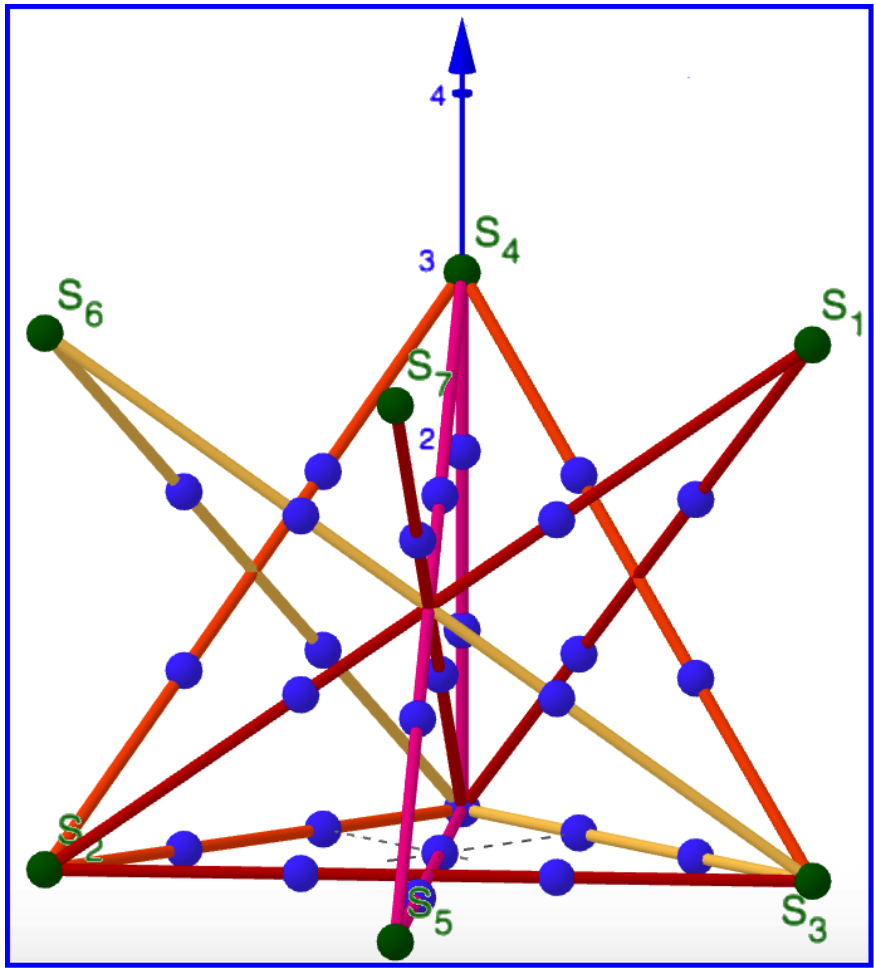

Figure 2. The extended clockwise-algorithm for the $3^{3}$-points problem. Perspective 1 (picture realized with GeoGebra [14]). 


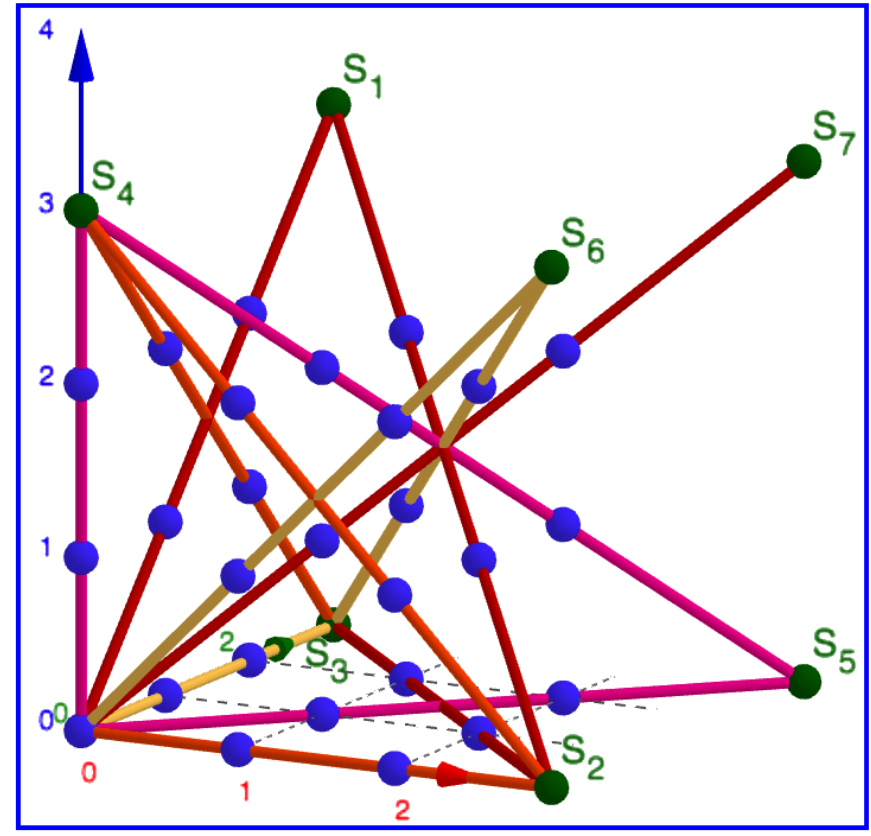

Figure 3. The extended clockwise-algorithm for the $3^{3}$-points problem. Perspective 2 (picture realized with GeoGebra [14]).

Theorem 1 Let $\tilde{h}_{n}$ be the Euclidean distance between the endpoints of the $n$-th link of the covering trail $H(k), \forall n, k \in \mathbb{N}-\{0\}$. If $L(k)=\{3 \cdot \sqrt{j}: j=1,2, \ldots,(k-1), k\}$, then $\left\{\tilde{h}_{1}, \tilde{h}_{2}, \ldots, \tilde{h}_{\left(\frac{3^{k}-3}{2}\right)}, \tilde{h}_{\left(\frac{3^{k}-1}{2}\right)}\right\} \subseteq L(k)$.

Proof. Let us invoke the standard clockwise-algorithm (briefly introduced in Section I), as described in Reference [8], which returns the covering trail $C(k)$. Thus, without loss of generality, we can follow $C(k)$ starting from any of its two endpoints (the clockwise-algorithm can never return a circuit). Then, let us call $\mathrm{P}_{1}$ the starting node, $\mathrm{P}_{2}$ any given Steiner point such that $\overline{\mathrm{P}_{1} \mathrm{P}_{2}}:=\frac{2}{3} \cdot \tilde{h}_{1}$, and so on until $\mathrm{P}_{\left(\frac{3^{k}+1}{2}\right)}$ such that $\overline{\mathrm{P}_{\left(\frac{3^{k}-1}{2}\right)} \mathrm{P}_{\left(\frac{3^{k}+1}{2}\right)}}:=\frac{2}{3} \cdot \tilde{h}_{\left(\frac{3^{k}-1}{2}\right)}$ is reached. With the only exception of $\mathrm{P}_{1}$ and $\mathrm{P}_{\left(\frac{3^{k}+1}{2}\right)}$, every $\mathrm{P}_{1<i<\left(\frac{3^{k}+1}{2}\right)}$ belongs to $B_{k}$.

Since the Euclidean distance between any pair of opposite vertices of a $k$-cube with edge $l=3$ units is given by $l \cdot \sqrt{k}$, it follows that the Euclidean distance between any pair of distinct elements of $B_{k}$ is $3 \cdot \sqrt{j}$, for some integer $j \in[1, k]$. Consequently, $\overline{\mathrm{P}_{l} \mathrm{P}_{(l+1)}}$ belongs to $L(k)$ for any $1<i<\left(\frac{3^{k}+1}{2}\right)$, and so does $\tilde{h}_{j}$ if $1<j<\left(\frac{3^{k}-1}{2}\right)$.

The only two remaining cases concern $\overline{\mathrm{P}_{1} \mathrm{P}_{2}}$ and $\overline{\mathrm{P}_{\left(\frac{3^{k}-1}{2}\right)} \mathrm{P}_{\left(\frac{3^{k}+1}{2}\right)}}$, but the extended clockwisealgorithm differs from the standard version for the prolongation (add $\frac{1}{3} \cdot \tilde{h}_{1}$ ) of the first link backward until we reach one point in $B_{k}$, and so also if we extend forward (add $\frac{1}{3} \cdot \tilde{h}_{\left(\frac{3^{k}-1}{2}\right)}$ ) the 


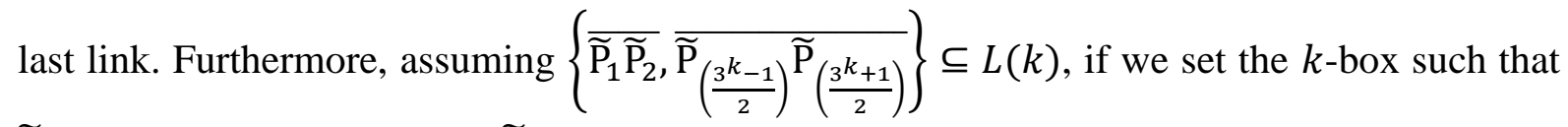
$\widetilde{\mathrm{P}}_{1} \equiv 0_{k}=(0,0, \ldots, 0)$, then $\widetilde{\mathrm{P}}_{\left(\frac{3^{k}+1}{2}\right)} \equiv(3,3, \ldots, 3)$ by construction.

Since $\quad \mathrm{P}_{i} \in B_{k} \quad \forall i \in\left\{1,2, \ldots,\left(\frac{3^{k}-1}{2}\right),\left(\frac{3^{k}+1}{2}\right)\right\}, \quad$ we have shown that, $\forall j \in$ $\left\{1,2, \ldots,\left(\frac{3^{k}-3}{2}\right),\left(\frac{3^{k}-1}{2}\right)\right\}, \tilde{h}_{j}$ belongs to $L(k)$, and this concludes the proof of Theorem 1 .

\section{INSIDE THE BOX COVERING PATHS WITH $\sqrt{5}$ UNITS LONG EDGES}

This section is devoted to prove that, $\forall k \in\{2,3\}, G_{k}$ can be covered inside the minimal $k$ dimensional box, $\hat{B}(k)$, by a simple path $M(k) \subset \widehat{B}(k)$ of link length $m(k)<3^{k}$, whose all $m(k)$ links belong to the unique length class $\sqrt{5}$.

Definition 3 Let us define, $\forall k \in \mathbb{N}-\{0\}, \hat{B}(k):=\left\{\left(x_{1}, x_{2}, \ldots, x_{k}\right): x_{1}, x_{2}, \ldots, x_{k} \in[0,2]\right\}$ $\subset \mathbb{R}^{k}$ as minimal-k-box.

Definition 4 Let $M(k) \subset \hat{B}(k)$ be a possibly self-intersecting simple path going through every element of the finite set $G_{k}$ (i.e., a covering path that visits the nodes of $G_{k}$ exactly once).

Theorem 2 If $k \in\{2,3\}$, then $\exists M(k) \subset \hat{B}(k)$ such that the Euclidean distance between the endpoints of all the $m(k)<3^{k}$ edges of $M(k)$ is $\sqrt{5}$.

Proof. We constructively prove Theorem 2 showing the existence of the two covering paths $M(2) \subset \widehat{B}(2)$ and $M(3) \subset \hat{B}(3)$, whose $m(k)=3^{k}-1$ edges belong, to the length class $\sqrt{5}$. If $k=2$, then $M(2)=(1,2)-(2,0)-(0,1)-(2,2)-(1,0)-(0,2)-(2,1)-(0,0)-\left(\sqrt{\frac{5}{2}}, \sqrt{\frac{5}{2}}\right)$ is a self-intersecting inside the box covering path with $\sqrt{5}$ units long edges that joins the 9 nodes of $G_{2}$. If $k=3$, then $M(3)=(2,0,0)-(0,1,0)-(2,2,0)-(1,0,0)-(0,2,0)-(1,2,2)-(0,0,2)$ $-(2,1,2)-(0,2,2)-(1,0,2)-(2,2,2)-(0,1,2)-(2,0,2)-(2,2,1)-(0,1,1)-(2,0,1)-(1,2,1)-$

$$
\begin{aligned}
& -(0,0,1)-(2,1,1)-(0,2,1)-(1,0,1)-(1,2,0)-(1,1,2)-(2,1,0)-(0,0,0)-\left(\sqrt{\frac{5}{2}}, \sqrt{\frac{5}{2}}, 0\right)- \\
& \left.-\left(\frac{\sqrt{10}}{12} \cdot(6-\sqrt{24-3 \cdot \sqrt{10}}), \frac{\sqrt{10}}{12} \cdot(6-\sqrt{24-3 \cdot \sqrt{10}}), \frac{1}{2} \cdot \sqrt{\frac{5}{3} \cdot(4+\sqrt{10}}\right)\right) \text { is a self- }
\end{aligned}
$$

intersecting covering path, with $\sqrt{5}$ units long edges, that visits the $27=m(3)+1$ nodes of $G_{3}$, without going outside the minimal-3-box (as shown in Figures $4 \& 5$ ). 


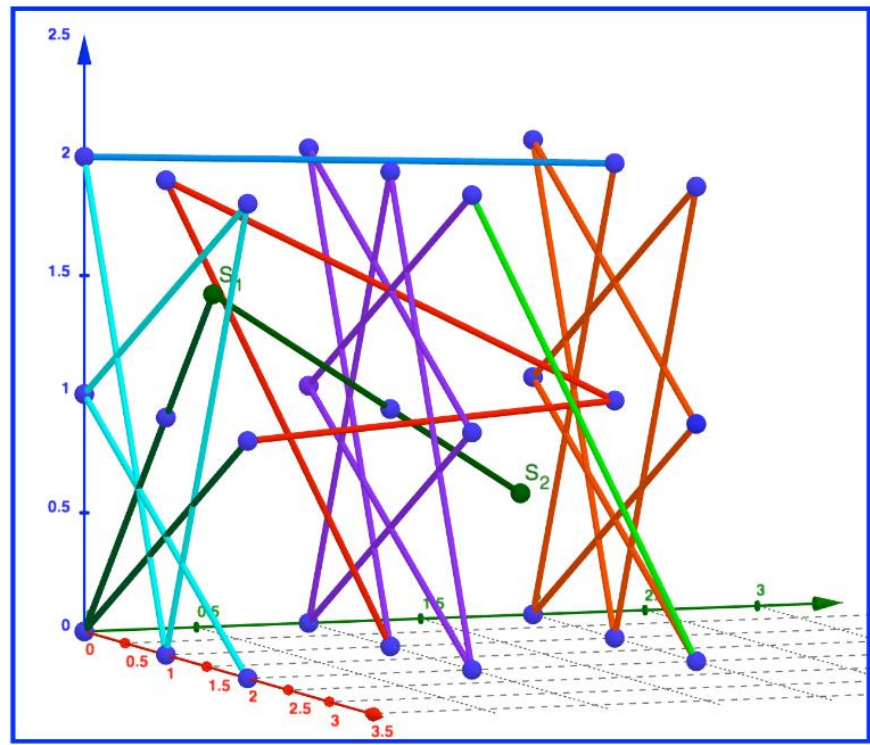

Figure 4. The covering path $M(3)$ consists of 26 edges, all belonging to the length class $\sqrt{5}$; the two Steiner points (in green) are $S_{1} \equiv\left(\sqrt{\frac{5}{2}}, \sqrt{\frac{5}{2}}, 0\right) \cong(1.58113883,1.58113883,0)$ and

$$
\mathrm{S}_{2} \equiv\left(\frac{\sqrt{10}}{12} \cdot(6-\sqrt{24-3 \cdot \sqrt{10}}), \frac{\sqrt{10}}{12} \cdot(6-\sqrt{24-3 \cdot \sqrt{10}}), \frac{1}{2} \cdot \sqrt{\frac{5}{3} \cdot(4+\sqrt{10})}\right) \cong
$$

(0.57721711, 0.57721711, 1.72750756). Perspective 1 (picture realized with GeoGebra [14]).

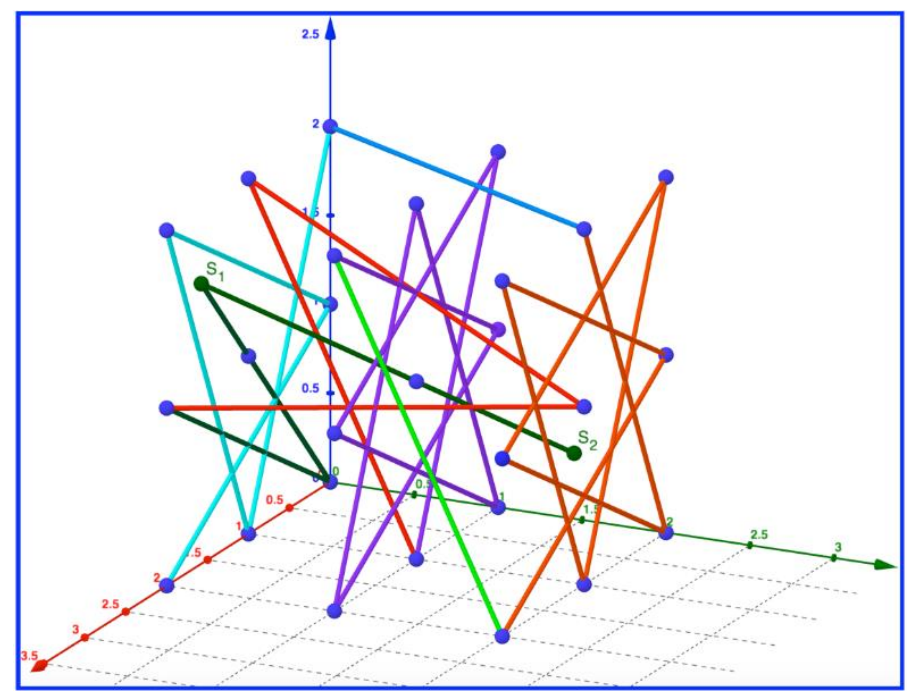

Figure 5. All the edges of $M(3)$ are entirely contained in the minimal-3-box $\widehat{B}(3)$. Perspective 2 (picture realized with GeoGebra [14]).

Corollary 1 An incomplete knight's tour of length 25 is possible on the $3 \times 3 \times 3$ board.

Proof. Referring to the well-known knight's tour problem [9, 10], it is trivial to point out how edges 1-24 of the covering path $M(3)$ (see proof of Theorem 2) provide also an incomplete open knight's tour of length 25 on the $3 \times 3 \times 3$ chess board. 
Since in 2007 Demaio [11] proved that it is impossible for the knight of the problem to visit every square of the $3 \times 3 \times 3$ board, $M(3)$ implies that the longest achievable sequence of edges having both their endpoints belonging to $G_{3}$ has a length of 25 or $26[12,13]$.

\section{CONCLUSION}

The $3^{k}$-points problem can be optimally solved (i.e., using the minimum number of links) with the covering trail $H(k)$ of link length $\frac{3^{k}-1}{2}$, and this result can be achieved through the standard clockwise-algorithm introduced in Reference [8] or using the extended clockwisealgorithm described in the present paper.

Considering the Euclidean distance between any two endpoints of the $\frac{3^{k}-1}{2}$ links generated by the extended version of the aforementioned algorithm, Theorem 1 shows that it produces no more than $k$ distinct length classes. Moreover, the extended clockwise-algorithm solves the enhanced $3^{k}$-points problem starting, ending, and turning only on the vertices of the $k$-box.

Therefore, every turning point is also a Steiner point that does not belong to $G_{k}$, with the only exception of the origin: the point $\mathrm{O}_{k}=(0,0, \ldots, 0)$, which can be (equivalently) taken as the starting/ending point of $H(k)$.

When we move the constraints on the number of the allowed length classes and on the volume of the $k$-box to the top of the list (rather than focusing ourselves on the link length of the covering trails as above), we face the NP-complete problem of finding Hamiltonian paths on $k$-dimensional grid graphs. For $k=2$ and $k=3$, Theorem 2 shows the existence of the covering paths $M_{\sqrt{5}}(k) \subset \hat{B}(k)$ whose $m(k)=3^{k}-1$ edges all belong to the length class $\sqrt{5}$, but different solutions are also possible (e.g., it is sufficient to take into account the set of uncrossing covering paths $M_{2}(k) \subset \hat{B}(k)$ for $G_{k}$, whose $3^{k}-2$ edges all belong to the length class 2 , which we are going to describe in another paper introducing the so-called "M $\mathrm{MI}$ algorithm").

\section{REFERECES}

[1] B. Chitturi and J. Pai, "Minimum-Link Rectilinear Covering Tour is NP-hard in R^4", arXiv, 1 Oct. 2018. https://arxiv.org/abs/1810.00529

[2] J. Minghui, "On covering points with minimum turns", International Journal of Computational Geometry and Applications, vol. 25, pp. 1-9, 2015.

[3] J. Wang, P. Tan, J. Yao, Q. Feng and J. Chen, "On the minimum link-length rectilinear spanning path problem: complexity and algorithms", IEEE Transactions on Computers, vol. 63, no. 12, pp. 3092-3100, 2014.

[4] B. Keszegh, "Covering Paths and Trees for Planar Grids", arXiv, 3 Nov. 2013. https://arxiv.org/abs/1311.0452

[5] J. M. Chein, R. W. Weisberg, N. L. Streeter and S. Kwok, "Working memory and insight in the nine-dot problem", Memory \& Cognition, vol. 38, pp. 883-892, 2010.

[6] M. Kihn, “Outside the Box: The Inside Story”, FastCompany, 1995. 
[7] S. Loyd, "Cyclopedia of Puzzles”, The Lamb Publishing Company, p. 301, 1914.

[8] M. Ripà, "Solving the 106 years old 3^k Points Problem with the clockwise-algorithm", Journal of Fundamental Mathematics and Applications, vol. 3, no. 2, pp. 84-97, 2020.

[9] A. Conrad, T. Hindrichs, H. Morsy and I. Wegener, "Solution of the Knight's Hamiltonian Path Problem on Chessboards", Discrete Applied Mathematics, vol. 50, no. 2, pp. 125-134, 1994.

[10] L. Euler, "Mémoire de Berlin for 1759”, Berlin, pp. 310-337, 1766.

[11] J. Demaio, "Which Chessboards have a Close Knight's Tour within the Cube", The Electronic Journal of Combinatorics, vol. 14, \#R32, p. 3, 2007.

[12] J. Erde, B. Golénia and S. Golénia, "The closed knight tour problem in higher dimensions", The Electronic Journal of Combinatorics, vol. 19, no. 4, \#P9, 2012.

[13] A. Kumar, "Magic Knight's Tours in Higher Dimensions", arXiv, 2 Jan. 2012. https://arxiv.org/abs/1311.0452

[14] M. Hohenwarter, M. Borcherds, G. Ancsin, B. Bencze, M. Blossier, J. Elias, K. Frank, L. Gal, A. Hofstaetter, F. Jordan, B. Karacsony, Z. Konecny, Z. Kovacs, W. Kuellinger, E. Lettner, S. Lizelfelner, B. Parisse, C. Solyom-Gecse and M. Tomaschko, "GeoGebra - Dynamic Mathematics for Everyone - version 6.0.507.0-w", International GeoGebra Institute, 16 Oct. 2018. https : / / www . geogebra .org 